\title{
La soi-disant petite insuffisance hépatique
}

Dans nos pays européens de langue latine, on taxe volontiers de petite insuffisance hépatique, des manifestations digestives relevant soit d'allergie alimentaire chronique, soit de banale intolerance ali-mentaire. Faut-il vraiment rattacher ces manifestations à une insuffisance du foie, entendons à une déficience du parenchyme de cet organe? Le problème fut discuté l'an dernier au cours d'un symposium à Geneve et nous voudrions ébaucher ici le point de vue que nous y avons développé avec P. Leonard.

Notre position est nette: il n'est en aucune façon démontré qu'il existe chez ces patients une insuffisance cellulaire hépatique. Après des considerations sur $\Gamma$ allergie et $\Gamma$ intolérance alimentaires, nous toucherons au næud du problème, la part du foie.

La place de Tallergie alimentaire chronique chez un dyspeptique est très difficile à définir, et le diagnostic en est malaise, surtout chez Tadulte, en raison de la déficience des tests cutanés. Un argument de présomption revient à la coexistence de stigmates allergiques, particu-

406

\section{Editorial}

lièrement Tasthme, le rhume des foins et la rhinite vaso-motrice. Duchaine a dressé comme suit la liste des aliments allergéniques notes en Belgique par ordre d'importance décroissante: ceufs, froment, pommes de terre, fromages cuits, lait, fruits saisonniers.

A côté de cette allergie alimentaire chronique avec conflit antigène-anticorps, probablement assez fréquente, on rencontre très commune-ment dans nos conditions de vie, ce qu'on peut appeler l'intolérance alimentaire banale.

Passant sous silence les boissons alcoolisées, cette intolerance commune porte principalement sur trois elements:

$1^{\circ}$ Des protéines dégradées, celles notamment des bouillons de viande. Elle est notée très souvent dans les diarrhées prandiales.

$2^{\circ}$ Les graisses et surtout les graisses cuisinées. Par ailleurs les graisses végétales sont parfois plus mal iolérées que le beurre frais. L'intolérance au chocolat est, on le sait, d'une extreme frequence.

$3^{\circ}$ Certaines formes de cellulose: pain complet, nombreux fruits, parfois legumes crus.

On a invoqué dans ces cas d'intolérance alimentaire, une insuffi-sance des secretions digestives; mais cette insuffisance sécrétoire reste mal mesurable. Soit dit en passant, la valeur digestive de la bile a été peu étudiée; ajoutons que le pancreas reste difficile à interroger dans sa function exocrine.

On connait le role aggravant d'un déséquilibre colique.

Un fait evident est $\Gamma$ existence d'un facteur constitutionnel, souvent familial, à predominance feminine; c'est dès Гenfance qu'existe fré-quemment une mauvaise tolerance à certains aliments, avec ou sans orages intermittents, comme migraines, vomissements périodiques, troubles intestinaux.

Classique encore l'intrication des manifestations d'intolérance avec le fonctionnement ovarien, bien souligné par Binet et de nombreux auteurs. 
De toute façon, de même que pour nous il existe un terrain aller-gique, Гintolérance alimentaire paraît s'intégrer dans le cadre d'une dystonie neuro-végétative ou neuro-endocrienne; la part très considerable des facteurs psychiques vient s'inscrire dans la même ligne.

Et le role du foie?

Sur le plan experimental, c'est dans le problème de 1'allergie qu'un jugement sur le role du foie peut être ébauché; et $\Gamma$ allergie étant un

Editorial

407

phénomène global, ce que nous savons du choc, grand accident aller-gique, s'applique toutes proportions gardées, à l'allergie alimentaire chronique. Nous bornant aux conclusions des experiences soit d'hépat-ectomie, soit de fistules d'Eck, schématiquement on peut dire que pour faire un choc, il faut un foie present et aussi un foie à functions nor-males, permettant les reactions vaso-motrices déclenchées par le conflit antigène-anticorps.

En clinique n'est-il pas frappant d'observer une disparition ou une diminution des explosions d'allergie dans les états d'insuffisance cel-lulaire marquee du foie, comme cirrhose décompensée ou dans le cours de l'hépatite infectieuse?

Nous avons pratique de nombreux tests de floculation, tant chez des allergiques que dans Tintolérance alimentaire commune, et trouvé une réponse le plus souvent norniale. Turiaf faisait récemment la même constatation chez les asthmatiques.

Une donnée intéressante est Faction fréquemment suspensive de la grossesse sur les manifestations allergiques. Pelner croit qu'il faut incriminer une authentique insuffisance du foie au cours de la gravi-dité. Pour le même auteur, c'est à un effet dépresseur sur les fonctions hépatiques qu'il faut attribuer Faction amortissante, efficacement notée en clinique du déhydrocholate de soude sur les manifestations allergiques.

La clinique plaide done, comme Fexpérimentation, pour la nécessité dans le déclenchement des accidents allergiques, d'un foie present et d'un foie dont les fonctions sont actives, voir hyperactives.

Nos conclusions rejoignent notre point de depart.

Dans Fintolérance alimentaire banale, comme dans l'allergie alimentaire chronique, une insuffisance cellulaire hépatique n'est nulle-ment apparente.

Bien plutôt, les phénomënes cl·miques d'allure hépatique ou biliaire apparaissent comme réactionnels, et on devrait parler de dyshépatie, voir d'hyperhépatie. Ces manifestations d'irritabilité au niveau du foie sont comtemporaines de celles que Fon observe dans le tube digestif proprement dit, ou encore dans d'autres secteurs de l'économie. J. Massion, Bruxelles 